\title{
approche probabiliste et géostatistique de la notion de porosité fissurale
}

\author{
par \\ A. Thomas \\ Tectonophysicien \\ Chargé de Recherche CNRS \\ E. N.S. Géologie de Nancy \\ A. Pineau \\ Mécanicien des Roches \\ Assistante à l'ENSG \\ P. Richard \\ Ingénieur Physicien \\ ENSG-INPL
}

\section{Introduction}

Tous les modèles traitant du mouvement des fluides dans les milieux poreux et une partie des modèles mécaniques de tels milieux, font référence au paramètre porosité classiquement défini comme le rapport $\ell=U \mathrm{p} / \mathrm{u}$ du volume Up des vides dans un volume u de mesure.

II est évident que cette définition est insuffisante si la porosité dépend du volume de mesure, comme c'est le cas général pour la porosité fissurale.

Cornet (1) propose une définition de la porosité comme fonction scalaire d'une variable vectorielle et analyse successivement :

- la porosité linéaire;

- la porosité de surface;

- la porosité volumique.

Nous verrons que certains aspects de cette définition peuvent légitimement être repris pour la porosité fissurale, mais que les caractères spécifiques des réseaux de fissures ou fractures vont nous obliger à abandonner certaines hypothèses telles que la stationnarité, ce qui nous amènera à considérer les porosités linéaire, de surface et volumique en un point comme des fonctions de variables régionalisées, ce qui implique d'une part d'associer une probabilité à toute définition de la porosité dans un domaine, d'autre part de caractériser clairement l'interdépendance entre la porosité et les distributions de fissures dans le domaine de mesure.

\section{Porosité linéaire}

\subsection{Définition de F.H. Cornet}

Considérant 2 points $x$ et $y$ à l'intérieur d'un volume rocheux, Cornet (1) définit après Scheidegger (5) la porosité linéaire du vecteur $\mathrm{u}=\mathrm{x}-\mathrm{y}$ par :

$$
\ell(u, x)=\left|u_{p}\right| \cdot|u|-1
$$

où $\left|u_{p}\right|$ est la portion de $u$ appartenant à l'espace poreux.

Si $|u|=0$

$\{\ell(x, 0)=0$ si $x$ appartient à la matrice solide,

$\{\ell(x, 0)=1$ si $x$ appartient à l'espace poreux.

A partir d'une représentation approchée des variations, quand $|\mathrm{u}|$ augmente, de $\ell(\mathrm{x}, \mathrm{u})$ pour un point $\mathrm{x}$ situé à l'intérieur de la matrice solide, et pour un point $x^{\prime}$ de l'espace poreux au voisinage de $\times$ (fig. 1), il constate que pour des valeurs de u suffisamment grandes, les valeurs de $\ell(x, u)$ et de $\ell\left(x^{\prime}, u\right)$ deviennent très proches.

D’où une définition de la porosité linéaire en un point $x$ dans une direction $v$ : ( $v$ vecteur unitaire)

$$
\ell=\ell\left(\mathrm{x}, \mathrm{u}_{\mathrm{o}}\right)
$$

où $\mathrm{u}_{\mathrm{o}}=\mathrm{kv}$ est défini comme la valeur de $\mathrm{u}$ pour laquelle :

$$
\Delta \ell=\left|\ell\left(\mathrm{x}, \mathrm{u}_{\mathrm{o}}\right)-\ell\left(\mathrm{x}^{\prime}, \mathrm{u}_{\mathrm{o}}\right)\right| \leqslant \varepsilon .
$$

$\varepsilon$ étant choisi avec la précision voulue par l'investigateur.

Nous aborderons le problème fissural avec une approche un peu différente.

\subsection{Analyse des hypothèses}

La formulation précédente suppose plusieurs hypothèses implicites, en général légitimes pour la porosité matricielle mais qui méritent examen pour la fissuration.

La première hypothèse est que l'équation (2) que nous écrirons

$$
|\Delta \ell| \leqslant \varepsilon
$$

ne comporte qu'une solution.

La seconde est que la valeur de $u_{0}$ fournie par l'équation (2) est indépendante de la position de $\mathrm{x}$.

$\checkmark$ La troisième est que la valeur de $u_{0}$ ne dépend pas 


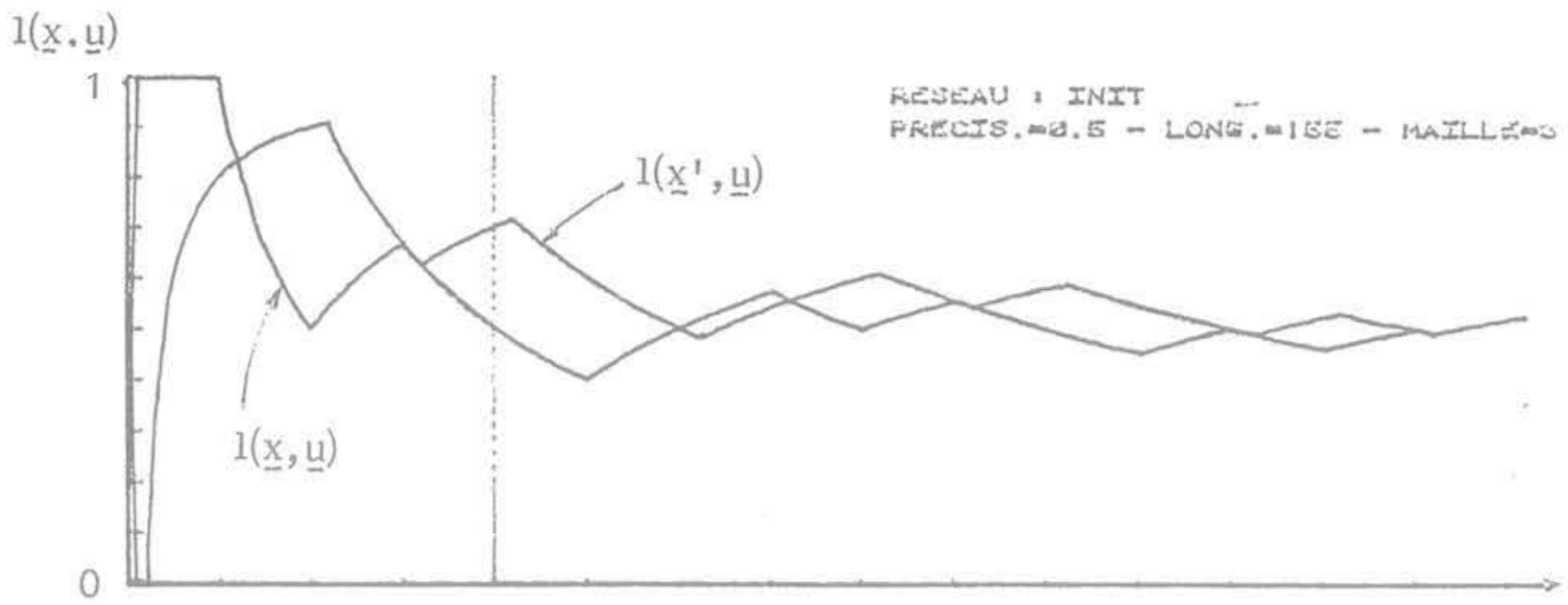

Fig. 1 Variation de $1(x, u)$ en fonction de $u$

de $\Delta x=\left|x-x^{\prime}\right|$ pourvu que celui-ci soit " suffisamment petit „, mais dépend de l'appartenance de $x$ et $x^{\prime}$ à des milieux différents.

- On voit sur la figure 1 que l'hypothèse (1) n'est légitime que si l'on raisonne sur une forme lissée de la fonction $I(x, u)$ ou sur une courbe-enveloppe. Elle est illégitime pour la courbe réelle.

- L'hypothèse (2) en sous-tend une autre qui est la stationnarité de la distribution des "vides" dans le champ d'investigation.

- L'hypothèse (3) est à rejeter si l'on ne postule pas que $\Delta x$ est très petit par rapport à un pore. La figure 2 , montre que :

- indépendamment des appartenances respectives de $\mathrm{x}$ et $\mathrm{x}^{\prime}$ à la matrice et aux vides, $\Delta \ell$ varie largement avec $\Delta x$ (courbes 3.4.5.);

- cette variation n'est amplifiée qu'au voisinage de l'origine de $u$ si $x$ et $x$ ' sont dans des milieux différents (courbes 3 et 4 ou 1 et 2 ).

Nous reformulerons donc la définition de la porosité linéaire en tenant compte des remarques faites précédemment.

Pour la porosité fissurale, il nous paraît essentiel de tenir compte de l'importante u instabilité " initiale liée à la valeur de $\Delta \mathrm{x}$, et d'autre part d'envisager le problème des distributions non stationnaires.

Pour celà, il est plus facile de considérer $\ell(x, u)$ comme le produit :

- de la fonction continue dérivable $f(u)=|u|^{-1}$;

- d'une fonction Up $(x, u)$ continue dérivable, mais dont la dérivée est un processus aléatoire discontinu (fig. 3).

La fonction Up $(x, u)$ est parfaitement définie par la donnée de cette dérivée :

$$
|\delta U p|=d|U p| / d|u| \text {. }
$$

$\left|\delta U_{0}\right|$ est une épreuve d'un processus aléatoire classique (fig. 3) prenant la valeur 0 et 1 par saut à des instants ou abscisses aléatoires : nous la désignerons par "densité de vides fissuraux".

Ceci posé, la porosité linéaire s'écrit donc :

$$
\ell(x, u)=\frac{1}{|u|} \int_{0}^{u}\left|\delta u_{p}\right| d u
$$

et cette intégrale est définie pour chaque épreuve de la fonction aléatoire $U \mathrm{p}_{\mathrm{x}}$. Puisque ce n'est autre que la moyenne sur $u$ de la densité de vides fissuraux $\delta \cup p_{x}$, au voisinage de l'origine $x$.

Puisqu'il s'agit d'une variable aléatoire exprimée sur un support spatial, $\delta U$ p peut être considéré comme une variable régionalisée dont $\ell(x, u)$ mesurée sur $u$ représente un estimateur $\delta \mathrm{Up}_{\mathrm{x}}$ de la moyenne réelle $\overline{\delta U p}_{x}$ supposée stationnaire dans le domaine de mesure "linéaire" $\mathrm{u}$, au voisinage de $\mathrm{x}$.

Rappelons que la stationnarité d'ordre 2, hypothèse de la géostatistique minière, suppose les 2 premiers moments invariants par translation sur l'espace de définition, en l'occurence :

$$
\begin{aligned}
& E[\delta U p(u)]=m \\
& E\left[\delta U_{p}(u+h)-\delta U p(u)^{2}\right]=2 \gamma(h) .
\end{aligned}
$$

Notre problème est de choisir un vecteur $u_{0}$ appartenant au domaine stationnaire $u$ tel que l'estimateur $\delta U p_{x}\left(u_{0}\right)=\ell\left(x, u_{0}\right)$ représente une estimation satisfaisante de la porosité cherchée $\delta U p$.

$2 \gamma(h)$ représente la variance d'estimation de $\delta U p\left(u_{0}+\right.$ h) par $\delta \cup p\left(u_{0}\right)$. II est clair que la moyenne $m$ dépend de la longueur $\mathrm{u}_{0}$ que laquelle elle est calculée et du choix de l'origine pour un $u_{0}$ donné. En revanche la variance d'estimation ne dépend que de $\mathrm{u}_{0}$ si $\delta U_{\mathrm{p}}$ est stationnaire.

On ne peut donc caractériser la variance de la porosité qu'en fonction d'un segment $u_{0}$ et le choix de ce segment doit être tel que l'estimation $\ell\left(x, u_{0}\right)=\delta U p\left(u_{0}\right)$ ne dépende pas, à un seuil de probabilité donné, du choix de $x$ dans le champ de mesure.

\subsection{Variations liées au choix de l'origine. Détermination d'un seuil}

Essayons d'abord de faire la part des variations liées au choix de l'origine dans un réseau supposé stationnaire ou pas. II s'agit donc d'apprécier la valeur au voisinage de l'origine de la quantité :

$$
\Delta \ell\left(\mathrm{x}, \mathrm{x}^{\prime}, \mathrm{u}\right)=\left|\ell(\mathrm{x}, \mathrm{u})-\ell\left(\mathrm{x}^{\prime}, \mathrm{u}\right)\right|
$$

lorsque $x$ et $x^{\prime}$, restant voisins, se situent l'un dans un pore, l'autre dans un pont.

Cette fonction a été représentée sur la figure 4 pour différents doublets $\left(\left(X_{5}, X_{1}\right)\right.$ de la figure $2 a$. 
(2a)

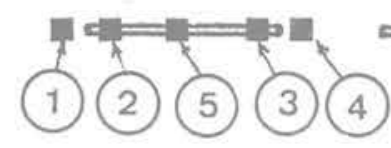

$l(x, u)$

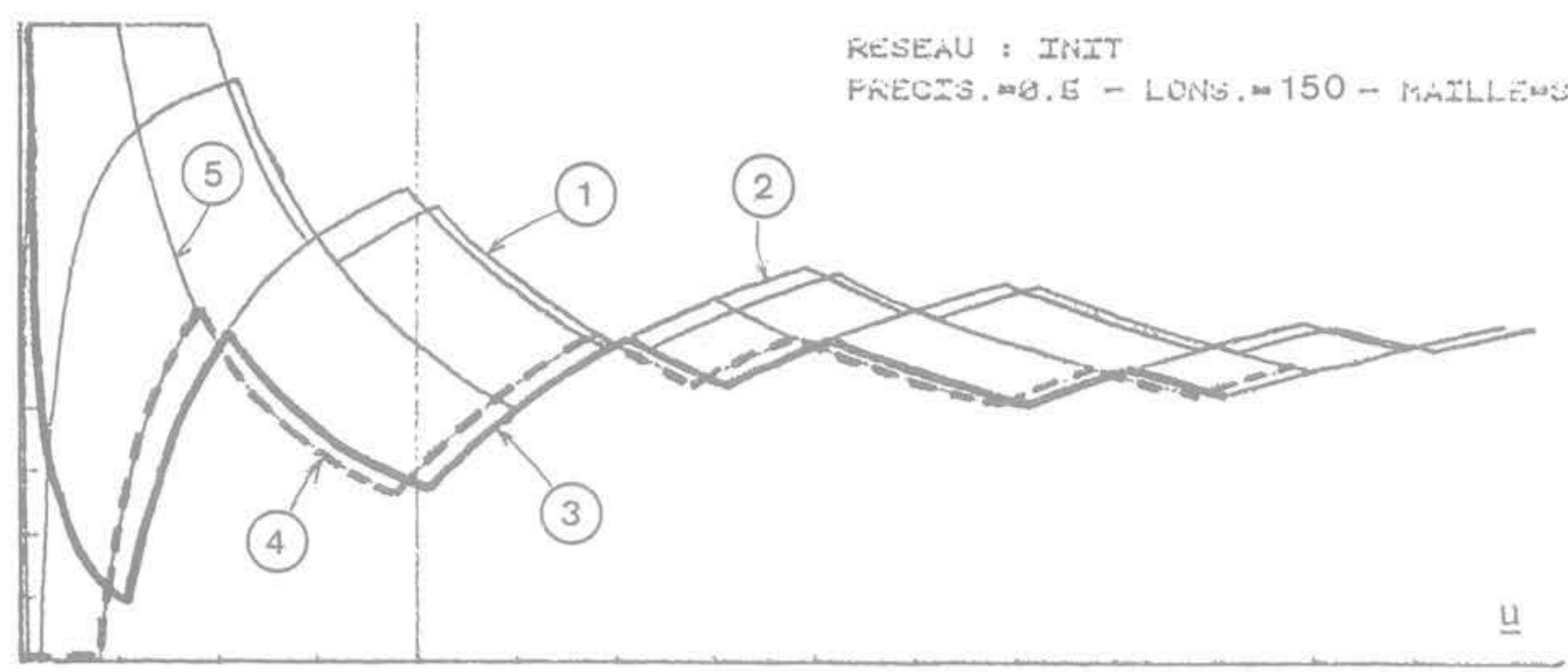

Fig. $2 a$

(2b)

0

Fig. $2 b$ variations de $1(x, u)$ pour différentes positions dans le réseau de la figure $2 a$ (ramenées à une origine commune)

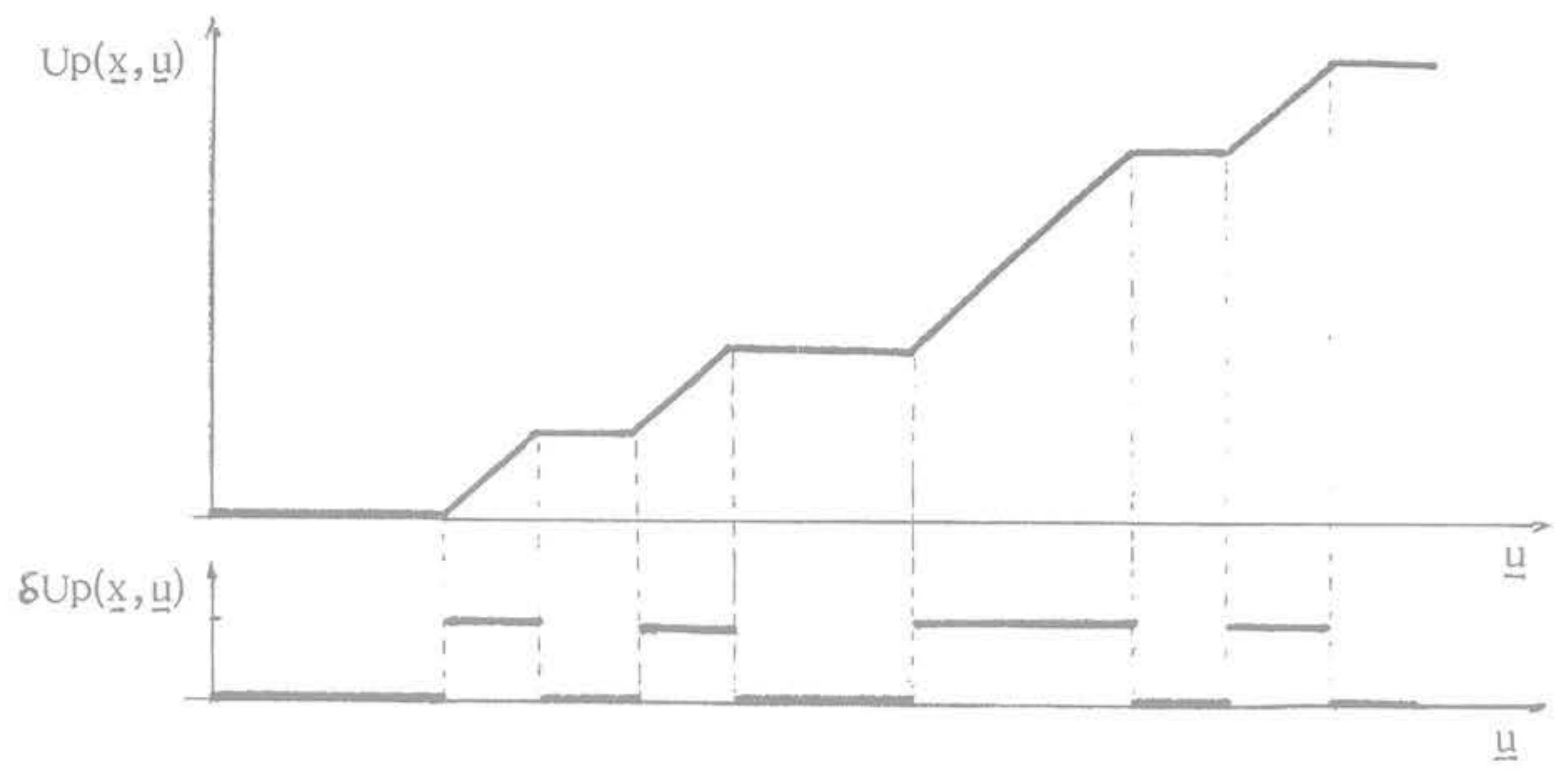

Fig. 3 Fonctions $U p(x, u)$ et $\delta U_{p}(x, u)$

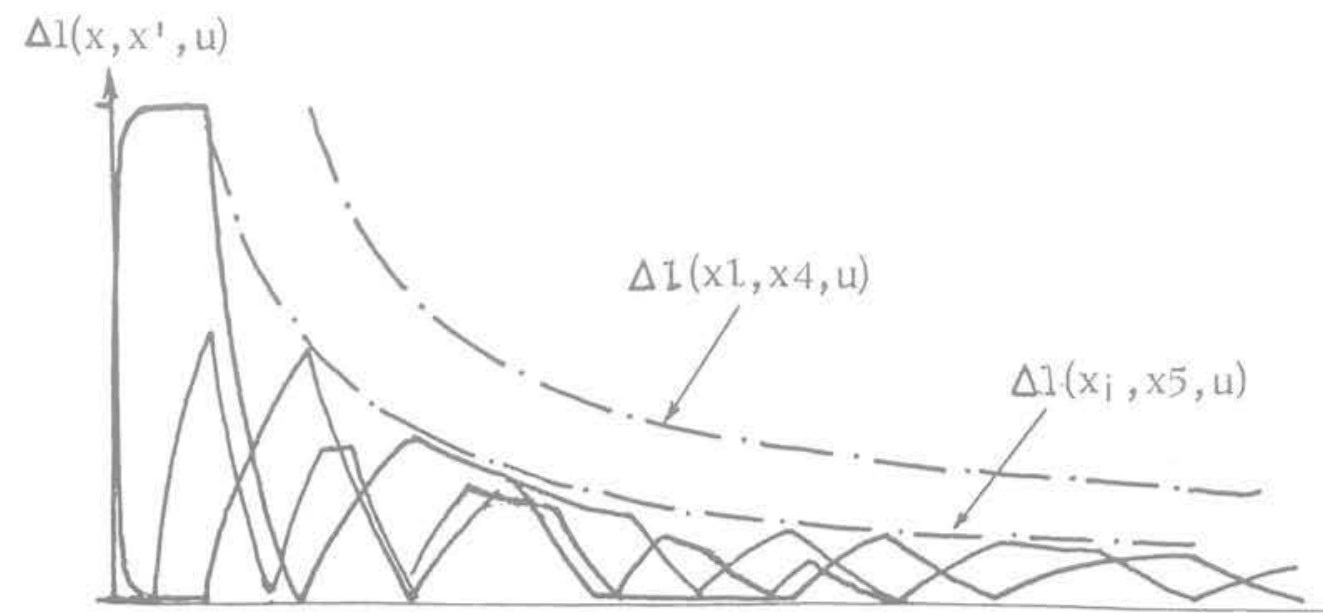

Fig. 4 Variation de $\Delta /\left(x, x^{\prime}, u\right)$ pour différents couples $\left(x, x^{\prime}\right)$ 


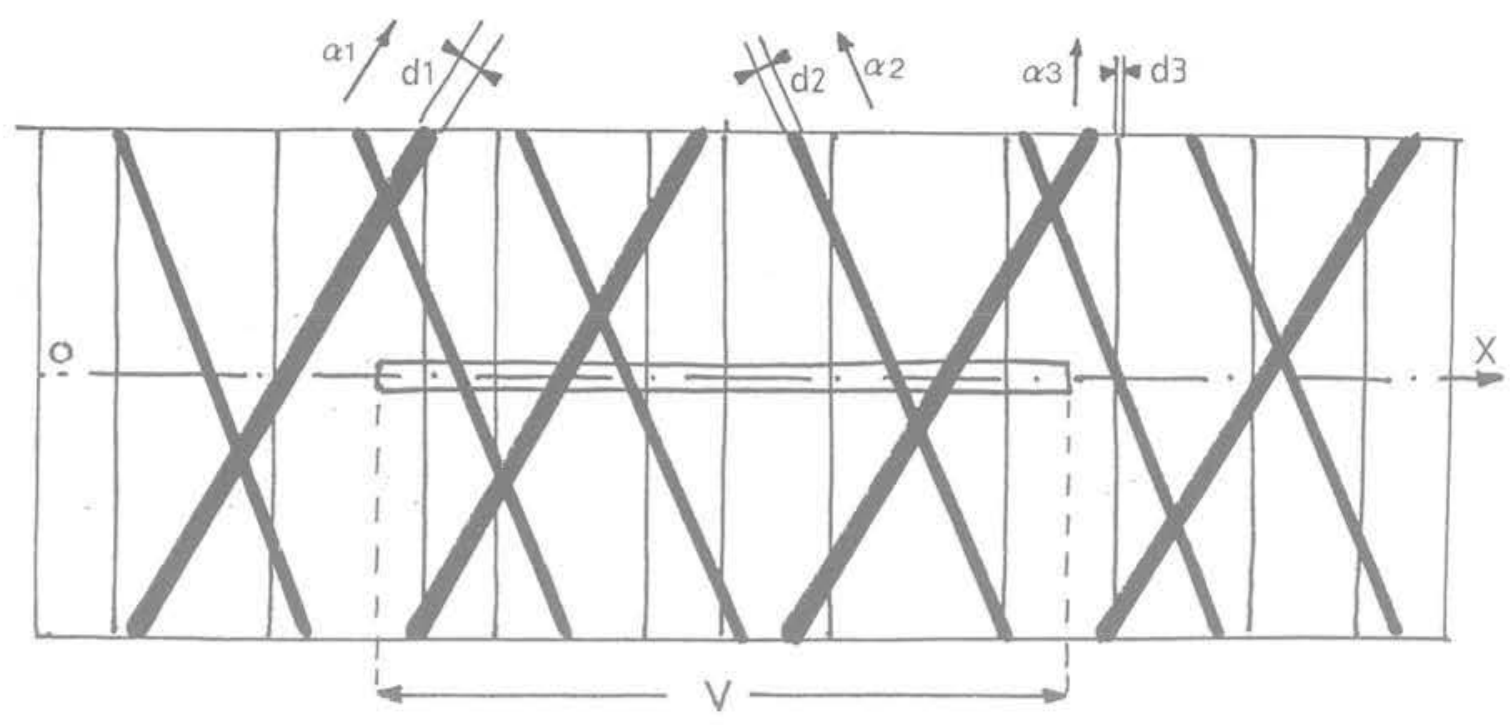

Fig. 5 Schéma de fracturation sur lequel est effectuée la mesure de porosité linéaire

II est bien clair que $\Delta \ell$ est limité supérieurement par la courbe enveloppe $\Delta \ell\left(x, x^{\prime}, u\right)$ définie par:

$$
\Delta \ell\left(\mathrm{x}, \mathrm{x}^{\prime}, \mathrm{u}\right)=\left|\mathrm{x}-\mathrm{x}^{\prime}\right| \cdot|\mathrm{u}|^{-1} \text {. }
$$

Si l'on admet que $x$ est choisi au hasard dans le champ d'investigation, entre $x^{\prime}$ et un point $x^{\prime \prime}$ de son voisinage dans le milieu différent contigu dans la direction de $u$, $\Delta l$ est borné supérieurement par la courbe :

$$
\Delta \ell \operatorname{Max}\left(\mathrm{x}^{\prime}, \mathrm{x}^{\prime \prime}, \mathrm{u}\right)=\operatorname{Max}\left|\mathrm{x}^{\prime}-\mathrm{x}^{\prime \prime}\right| \cdot|\mathrm{u}|^{-1}
$$

où $\operatorname{Max}\left|x^{\prime}-x^{\prime \prime}\right|$ représente la dimension du plus grand pore ou du plus grand pont sur l'espace accessible au vecteur $\left|x^{\prime}-x^{\prime \prime}\right|$.

Supposons donc que dans un champ $V$, on veuille échantillonner la porosité sur des segments de longueur u implantés au hasard. Le choix d'un seuil de variation nécessite la connaissance de la borne supérieure $\Delta \ell$ Max déterminée par le plus grand pore ou le plus grand pont qui n'est pas nécessairement pris en compte par l'échantillonnage.

Supposons que nous possédons, dans un plan (C) incluant le champ de mesure $V$ et grand par rapport à lui une statistique ayant permis de dégager 3 familles $f_{i}$ de fractures linéaires d'orientations respectivement $\alpha_{1}$, $\alpha_{2}, \alpha_{3}$, par rapport à un axe repère d'épaisseur $d_{1}, d_{2}$, $d_{3}$, distribuées chacune selon un processus Poissonnien de densité $\lambda_{1}, \lambda_{2}, \lambda_{3}$ connue.

\subsubsection{Détermination du plus grand pore en probabilité}

On sait calculer les probabilités des intersections de ce réseau avec le champ de mesure linéaire $\mathrm{V}$.

Les probabilités pour que $V$ soit recoupé par un nombre $n=N$ de fractures sont pour chaque famille $i$ :

$$
P_{i}(n=N)=\frac{\left(\lambda^{\prime} \mathrm{iV}\right)^{n} e^{-\lambda i v}}{n^{\prime}} \operatorname{avec} \lambda^{\prime} i=\lambda i \sin \alpha i
$$

le support de $\mathrm{V}$ étant pris comme axe origine.
Les probabilités pour que $V$ soit recoupé au moins par une fracture sont pour chaque famille:

$$
\begin{array}{ll} 
& P_{i}(n \geqslant 1)=1-P_{i}(n=0) \\
\text { donc : } & P_{i}(n \geqslant 1)=1-e^{-\lambda i v} .
\end{array}
$$

La largeur du pore correspondant est $\mathrm{Di}=\mathrm{di} / \sin \alpha \mathrm{i}$ si I'on prend le support de $\mathrm{V}$ comme axe origine.

Ainsi à l'ensemble des 3 familles de segments intersectés par $V$, peut-on associer la loi de probabilité discontinue de la figure 6 .

A un seuil de probabilité donné, on peut donc déterminer le plus grand pore probable dans $V$ : dans l'exemple précédent au seuil de $90 \%$ le plus grand pore probable a la dimension D1, au seuil de $75 \%$ le plus grand a la dimension D2.

\subsubsection{Détermination du plus grand pont en probabilité}

On peut disposer d'une statistique sur les intervalles ou user des relations probabilistes simples existant entre la distribution des intervalles et celle des fractures pour chaque famille.

La figure 7 montre en coordonnées gaussologarithmiques un exemple de 3 lois de distribution pour les intervalles relatifs aux 3 familles $f_{1}, f_{2}, f_{3}$.

La probabilité globale $P(D)$ d'un intervalle de dimension $D \geqslant d_{0}$ donnée est la convolution des probabilités $P 1(D), P 2(D), P 3(D)$. C'est une loi continue.

La probabilité du plus grand pore ou du plus grand pont est alors représentée par la courbe $P(D)$ de la figure 8 . Dans notre exemple :

- au seuil de $90 \%$ le plus grand élément probable est un pont de dimension $\mathrm{D}=\mathrm{D}_{\max }(90$;

- au seuil de $50 \%$ le plus grand élément est un pore de dimension $\mathrm{D}=\mathrm{D}_{\max }(50)$. 


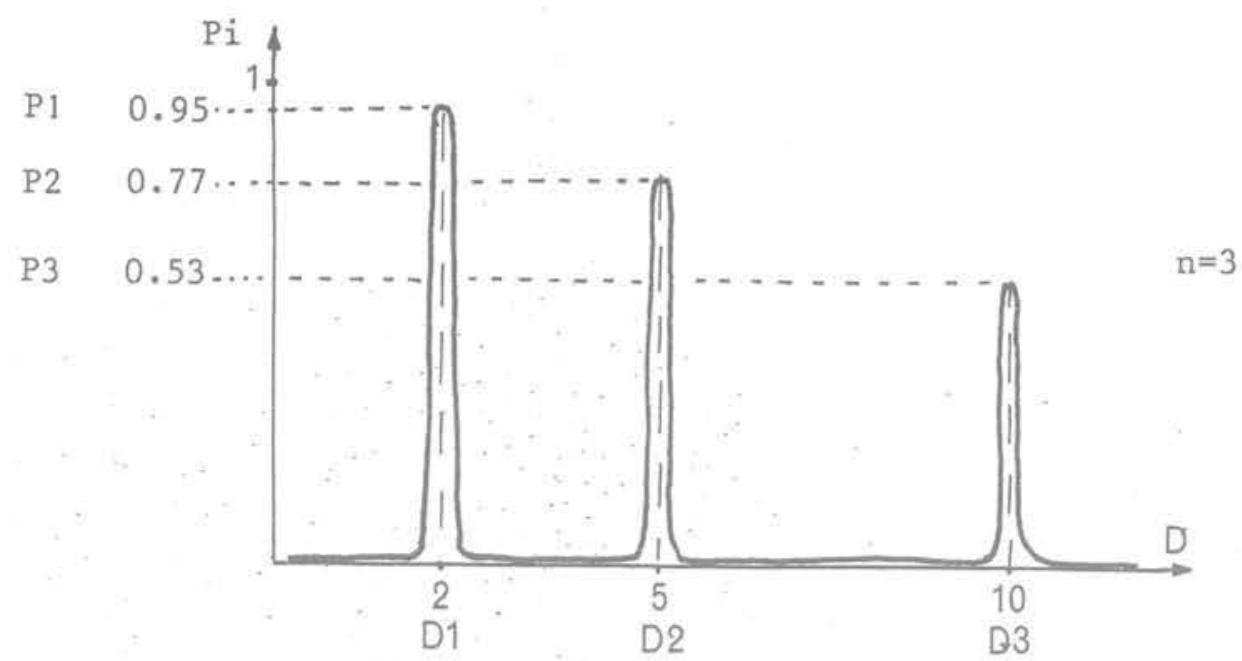

Fig. 6 Loi de probabilité discontinue Pi $(n \geqslant 1)=f(D i) \quad \lambda_{1}=1 \quad \lambda_{2}=0.5 \quad \lambda_{3}=0.25$

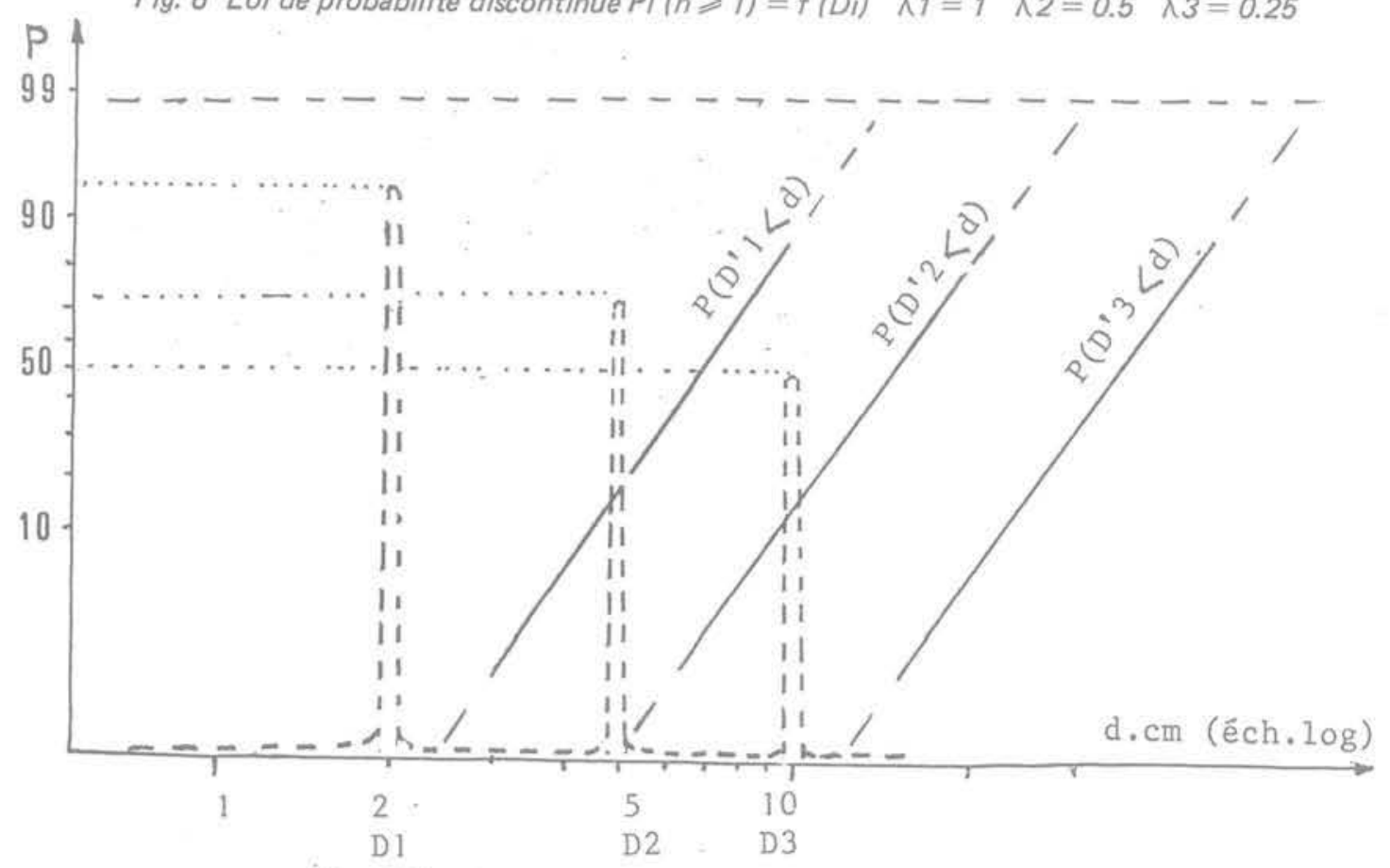

Fig. 7 Distribution des intervalles ou «ponts» pour $f_{1}, f_{2}, f_{3}$

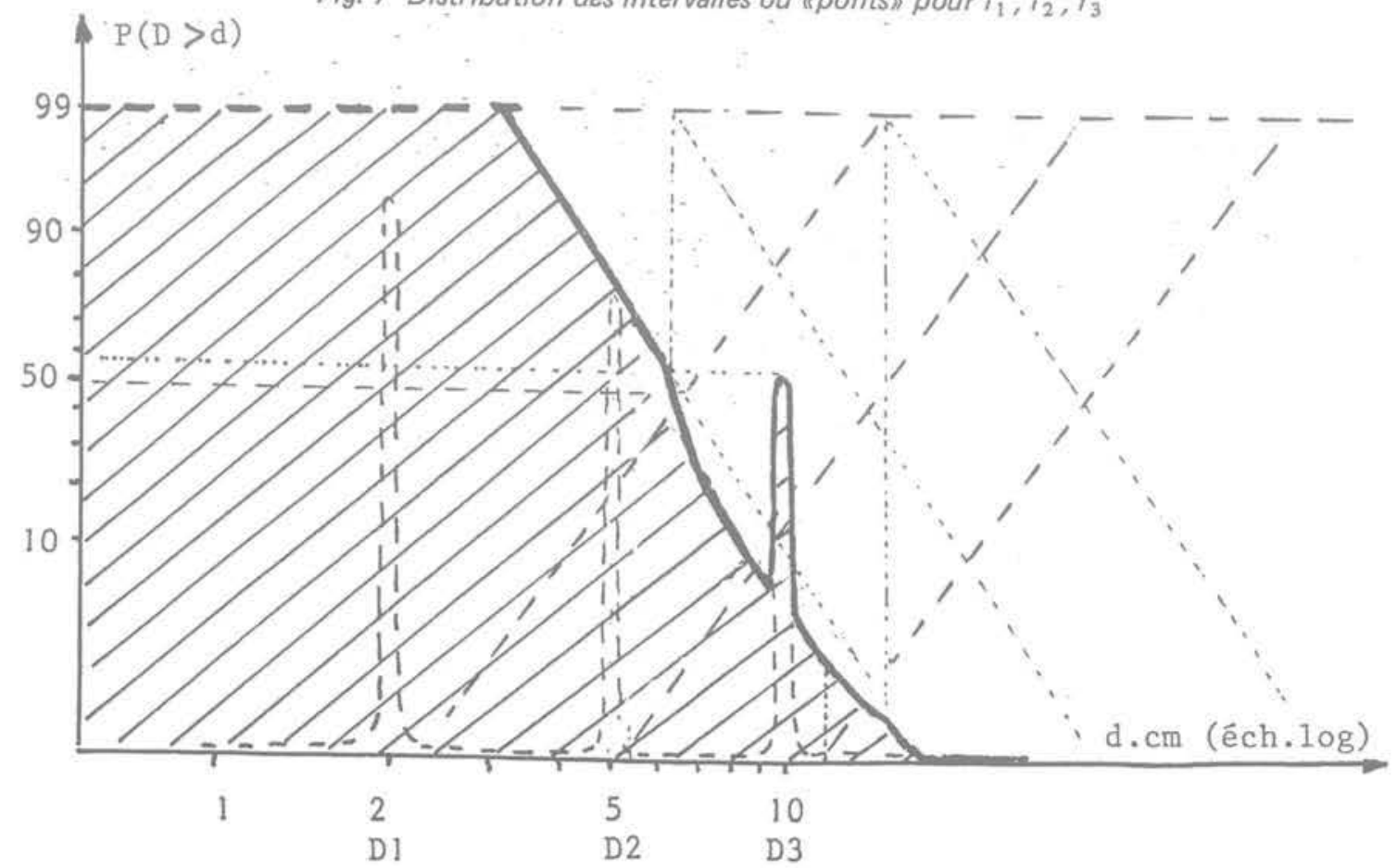

Fig. 8 Probabilité du plus grand pore ou du plus grand pont 


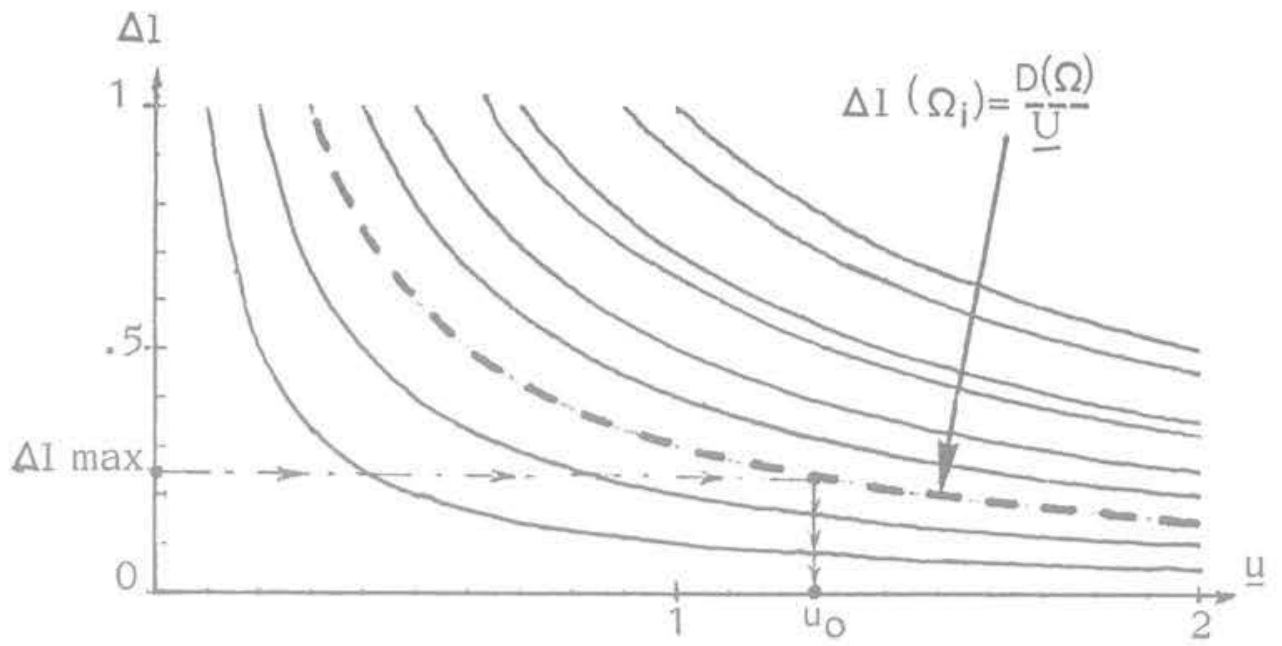

Fig. 9 Détermination de la longueur minimale Uo

\subsubsection{Détermination du seuil $u_{0}$}

Nous savons donc que $\Delta \ell(\mathrm{x}, \mathrm{u})$ est bornée supérieurement sur $V$ au seuil de $\Omega=90 \%$ par exemple, par la courbe :

$$
\Delta \ell_{\Omega}(\mathrm{x}, \mathrm{u})=\frac{\mathrm{D}_{\max }(\Omega)}{\mathrm{u}} .
$$

Le choix d'un écart maximum $\Delta \ell_{\max }$ sur la porosité donne, à ce seuil de probabilité, la valeur minimale $u_{0}$ sur laquelle doit être effectuée une mesure avec une origine prise au hasard sur $\mathrm{V}$ pour que l'ècart sur la porosité lié au choix de l'origine soit inférieur ou égal à $\Delta \ell_{\max }$ (fig. 9).

\subsection{Définition de la porosité}

La porosité échantillonnée au point $\mathrm{x}$ de $\mathrm{V}$ sera dans ces conditions le rapport

$$
\ell_{\Omega}(x, u)=\left|u_{p}\right| \cdot\left|u_{0}\right|^{-1}
$$

associé à la probabilité $\Omega$ et à l'erreur admissible $\Delta \ell_{\max }$. II est bien clair que la porosité ainsi définie est une fonction de $\Omega$ et $\Delta \ell_{\max }$ mais n'est plus fonction de $\mathrm{x}$ si elle est stationnaire sur V. II faudrait écrire :

$$
\ell\left[u\left(\Omega, \Delta \ell_{\max }\right)\right]=u_{p} / u_{0}\left(\Omega, \Delta \ell_{\max }\right) .
$$

Essayons de préciser la valeur de cette estimation ponctuelle de la porosité lorsque l'on se déplace dans le champ $V$.

La figure 10 montre l'ensemble, ramené à la même origine, des courbes de porosité obtenues sur le réseau figuré ( $Y Y 750)$ de $7,50 \mathrm{~m}$ en échantillonnant tous les $40 \mathrm{~cm}$ sur une longueur minimum $u_{0}$, en direction horizontale sur un banc de calcaire Bajocien des environs de Nancy.

$u_{0}$ a été déterminé à partir du plus grand pore réel $(\Omega=1)$ observé sur $V: D_{\max }=21 \mathrm{~cm}$ pour une variation admissible de 0,1 sur la porosité :

$$
\mathrm{u}_{0}(1,21)=\frac{D_{\text {Max }}}{\Delta \ell_{\max }}=\frac{21}{.1}=210 \mathrm{~cm} .
$$

L'échantillonnage de la porosité telle que nous l'avons défini est donc représenté par l'intersection des courbes avec la droites $u=u_{0}$.

Cet échantillon nous donne une certaine dispersion de la porosité. Notre problème est maintenant d'analyser la variance de cette dispersion dans le champ de mesure. Nous utiliserons pour celà la variance d'estimation de $\ell\left(x+h, u_{0}\right)$ par $\ell\left(x, u_{0}\right)$ lorsque $x$ se

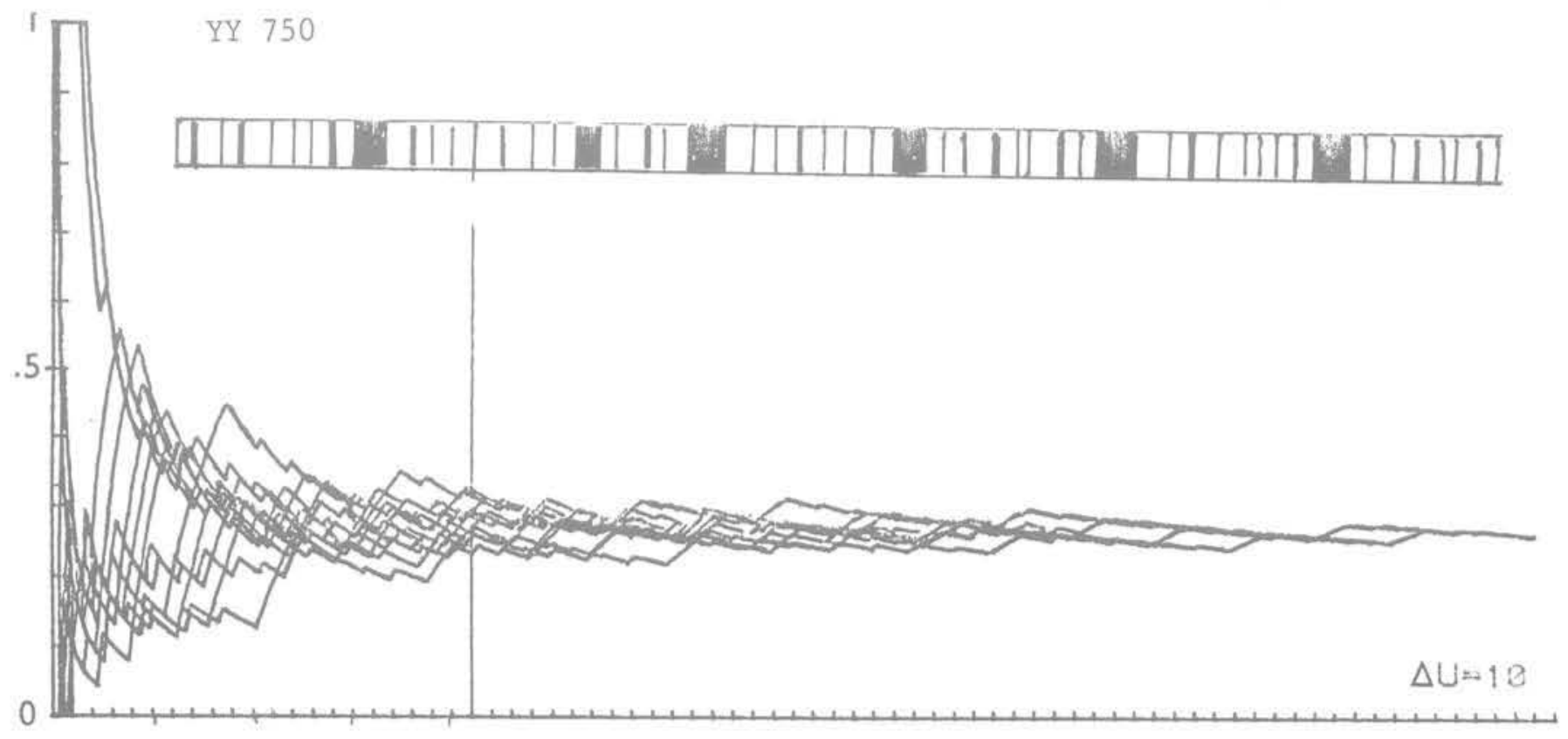

Fig. 10 Diagrammes de porosité d'un réseau gigogne à 2 familles 
déplace dans le champ, c'est-à-dire le variogramme, outil géostatistique qui nous permettra de tester la stationnarité et de déterminer à chaque échelle d'analyse la maille optimale d'échantillonnage de la porosité $\mathrm{I}\left(\mathrm{u}_{\mathrm{o}}\right)$ qui minimise la variance d'estimation.

\subsection{Analyse de la variance. Test de stationnarité}

Les deux outils classiques de cette analyse sont : $\sigma^{2}=E[\ell(x+h)-\ell(x)]^{2}$ variance d'extension ou variogramme

ou $(h)=\frac{1}{2} \sigma^{2}$ fonction intrinsèque ou demivariogramme

$\mathrm{C}(\mathrm{h})=\mathrm{E}[\ell(\mathrm{x}+\mathrm{h}) \cdot \ell(\mathrm{x})]-\mathrm{m}^{2}$ convariance centrée. Pour nous affranchir du biais lié à l'estimation simultanée de l'espérance $m$ et de la covariance non centrée, nous avons préféré comme il est d'usage en géostatistique, user du variogramme.

On se reportera à Serra (2.3.4) pour la compréhension de ce remarquable outil structural. Nous nous contenterons ici de rappeler :

que le comportement à l'origine caractérise les propriétés de continuité de la variable régionalisée (fig. 10) :

- comportement parabolique : variable à haute continuité $(10 \mathrm{a})$;

- comportement linéaire : régionalisations continues en moyenne;

- effet de "pépite " ; indiquant une régionalisation par une structure d'échelle inférieure à l'échelle d'analyse $(10 \mathrm{c})$;

- variable purement aléatoire : variance indépendante de l'espace (10d);

que le comportement à ul'infinin caractérise les "transitions " dans la régionalisation (fig. 11) :
- palier structural au-delà d'une "portée " p (fig. 12a) qui caractérise un phénomène stationnaire au-delà de cette portée (12b);

- structures gigognes caractérisées par l'existence de plusieurs paliers définissant chacun une échelle où le phénomène est stationnaire.

Notre propos n'est pas ici d'étudier des régionalisations précises de porosité mais de montrer l'intérêt du variogramme pour la définition de la porosité dans un champ $V$, et ceci que quelques exemples tirés d'une analyse de fracturation sur un front de taille du Bajocien Moyen à Nancy (France), analyse automatique effectuée selon une méthode originale de A. Thomas (6).

\subsubsection{Influence de $u_{0}$ sur la variance}

Les figures $13 \mathrm{~A}, \mathrm{~B}, \mathrm{C}$ et $\mathrm{D}$ représentent respectivement les variogrammes du réseau YY 750 correspondant à 4 valeurs de la tolérance $\Delta \mathrm{L}_{\mathrm{Max}}$ et donc 4 valeurs de $\mathrm{u}_{\mathrm{o}}$ pour un échantillonnage à maille $10 \mathrm{~cm}$.

L'amplitude $C$ de la variance varie fortement avec $\Delta \mathrm{L}_{\max }\left(\mathrm{ou} \mathrm{u}_{\mathrm{o}}\right.$ ) comme le montrent les valeurs de $\mathrm{C}_{\max }$ dans le tableau suivant:

\begin{tabular}{lcccc}
\hline & $A$ & $B$ & $C$ & $D$ \\
\hline$\Delta L_{\max }$ & 0,05 & 0,1 & 0,2 & 0,5 \\
$U_{0}(\mathrm{~cm})$ & 420 & 210 & 105 & 42 \\
$C_{\max } \times 10^{4}$ & 16 & 48 & 242 & 1468 \\
\hline
\end{tabular}

Pour analyser cette variation, nous avons reporté sur la figure 14, sur un même graphique les fonctions :

$$
\log \mathrm{C}=\mathrm{f}(\mathrm{h}) \text { pour les } 4 \text { valeurs de } \Delta \ell_{\max }\left(\mathrm{ou} \mathrm{u}_{\mathrm{o}}\right) \text {. }
$$
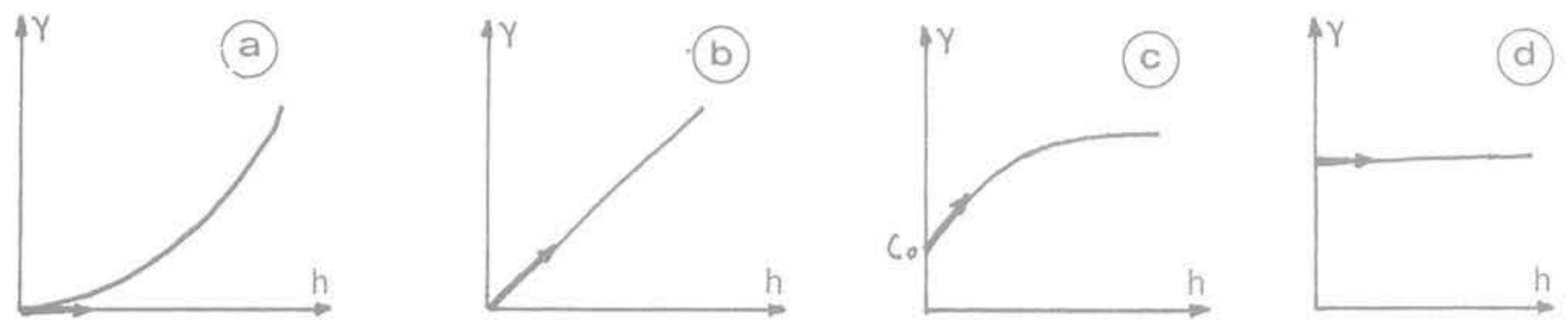

Fig. 11 Comportement à l'origine du variogramme (d'après SERRA)
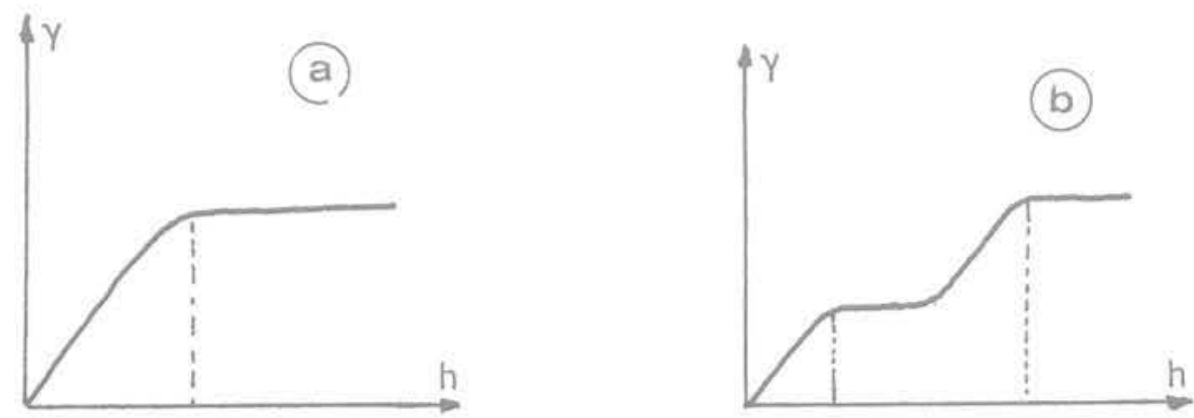

Fig. 12 Comportement à l'infini du variogramme (d'après SERRA) 


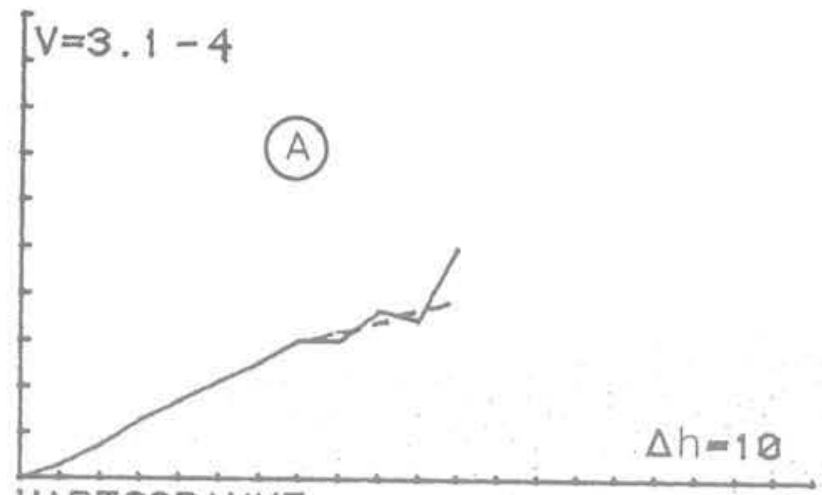

VARIOGRAMME

$\Delta L \max =0.05-M A I L L E \approx 10$

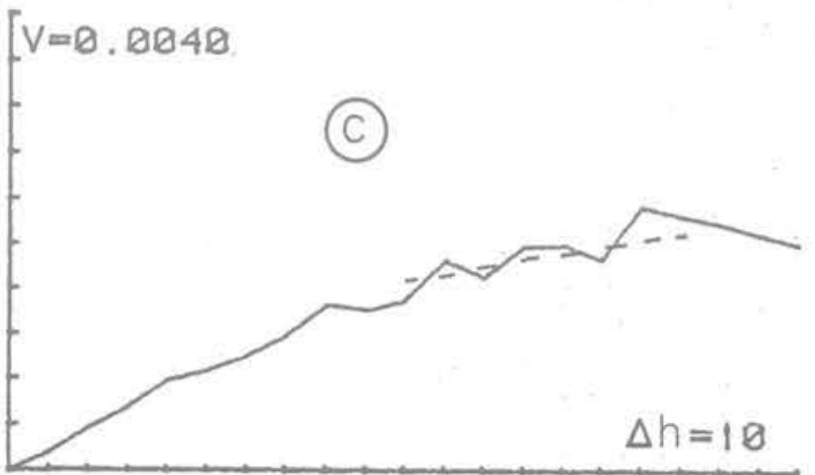

VARIOGRAMME

$\Delta \operatorname{Lmax}=0.2-$ MAILLE $=10$

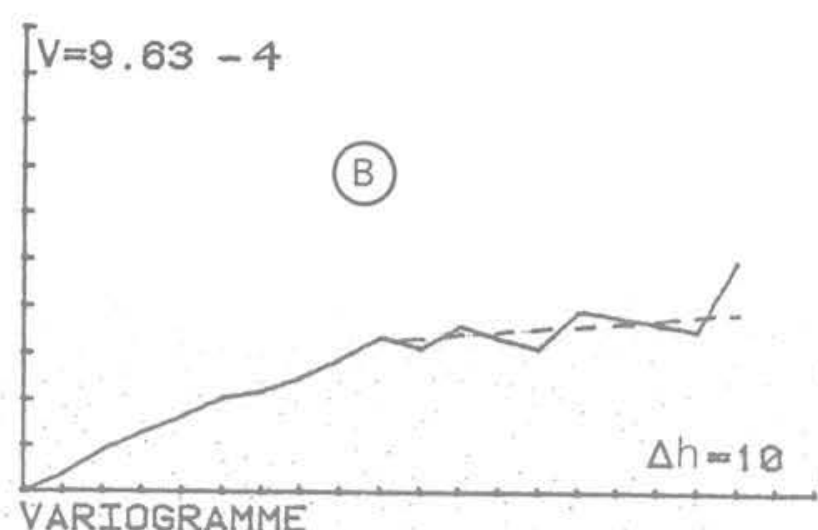

$\Delta L \max =0.1-$ MAILLE $=10$

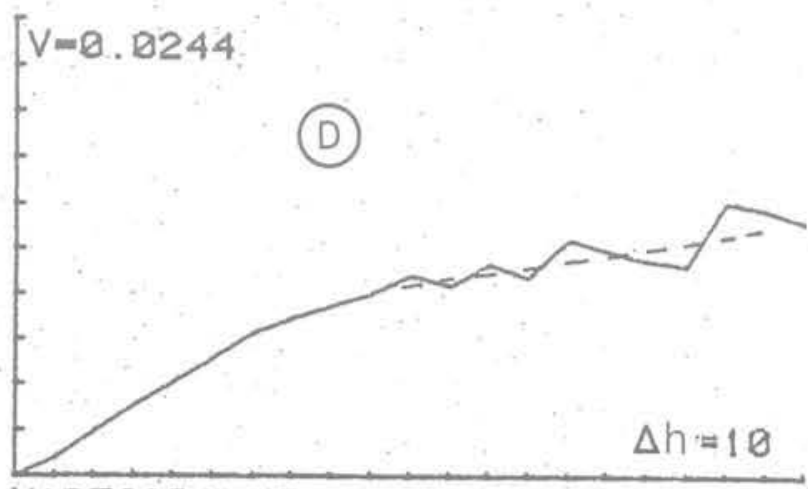

VARIOGRAMME

$\Delta L \max =0.5-$ MAILLE $=10$

Fig. 13 A, B, C, D : variogrammes du réseau YY750 à maille $100 \mathrm{~cm}$

Si nous utilisons la figure 14 pour construire la fonction $\log C=g(\Delta \ell)$ pour $h=190,110,40$ par exemple, on obtient les courbes de la figure 15 qui montrent que pour $\Delta \ell<0,2$, c'est-à-dire tant que $u_{0}>105$, la variation de $\log C$ avec $\Delta \ell$ est quasi linéaire. Cette loi varie avec $h$ pour les faibles valeurs de $h$ et se stabilise au voisinage de $h=100$.

Ceci signifie qu'une maille d'échantillonnage comprise entre 100 et 200 permet d'accéder à une porosité quasi stationnaire sur le champ, dont la variance ne dépend que de $u_{0}$ et de façon univoque. Fixer $u_{0}$ à partir de $\Delta \mathrm{L}_{\max }$ et d'une probabilité revient donc à fixer un seuil de variance à cette maille.

\subsection{Analyse structurale}

Essayons de préciser les caractères structuraux du réseau déductible des variogrammes lorsque varie la maille de réalisation de ces variogrammes.

On notera d'abord que, abstraction faite de l'amplitude $\mathrm{C}$ et donc à une affinité près, les caractères du variogramme ne varient pratiquement pas avec $u_{0}$.

En revanche, on constate en comparant respectivement les figures $13 \mathrm{~A}-16 \mathrm{~B}$ et $13 \mathrm{C}-16 \mathrm{~B}$ que l'information structurale varie avec la maille du variogramme. En particulier :

- les mailles $10 \mathrm{~cm}$ et $20 \mathrm{~cm}$ font apparaître une structure de dimension voisine de $80 \mathrm{~cm}$ matérialisée par un palier sensiblement stationnaire jusqu'à $200 \mathrm{~cm}$ (limite d'investigation). Cette structure correspond manifestement à la famille de grosses fractures.

A une maille d'échantillonnage inférieure à $80 \mathrm{~cm}$, cette structure échappe à l'investigateur comme le montre la partie quasi linéaire des courbes au voisinage de l'origine. Un léger " effet de pépite " laisse prévoir une structure d'ordre inférieur.

La figure 16 à maille $5 \mathrm{~cm}$ dégage en fait une double structure de dimension inférieure à $80 \mathrm{~cm}$ correspondant respectivement à des portées de 5 à $10 \mathrm{~cm}$ et de 25 à $30 \mathrm{~cm}$ qui doivent représenter les 2 générations de fissures les plus fines observables sur le réseau. L'amorce du palier à $80 \mathrm{~cm}$ y est visible également.

$\mathrm{Ce}$ variogramme met donc bien en évidence la structure gigogne de ce réseau sur lequel apparaît clairement qu'il existe plusieurs définitions possibles de la porosité, chacune correspondant à un domaine de stationnarité de la fonction $\ell(x, u)$.

II y a donc 3 échelles d'échantillonnage pour lesquelles on peut définir une porosité stationnaire dans le champ $\mathrm{V}$ :

- maille d'èchantillonnage de 5 à $20 \mathrm{~cm}$;

- maille d'échantillonnage de 30 à $50 \mathrm{~cm}$;

- maille d'échantillonnage de 80 à $200 \mathrm{~cm}$.

Le principe d'économie conduisant à choisir la plus grande maille à chaque échelle, les mailles optimales seront 20,50 et $200 \mathrm{~cm}$ selon que l'on désire voir la " carte de porosité " prendre en compte l'une ou l'autre de ces structures. 
$\mathrm{C} \times 10^{4}$

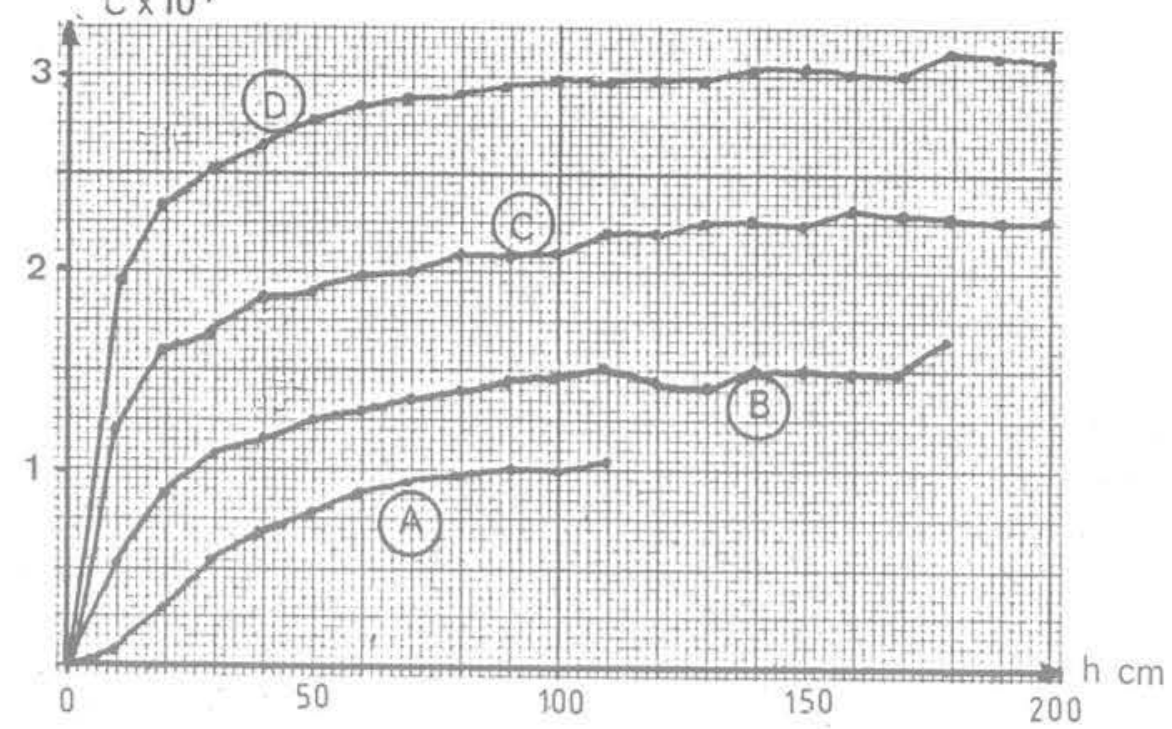

Fig. 14 Amplitude de la variance avec le pas "h» pour différentes valeurs de $\Delta / \max$

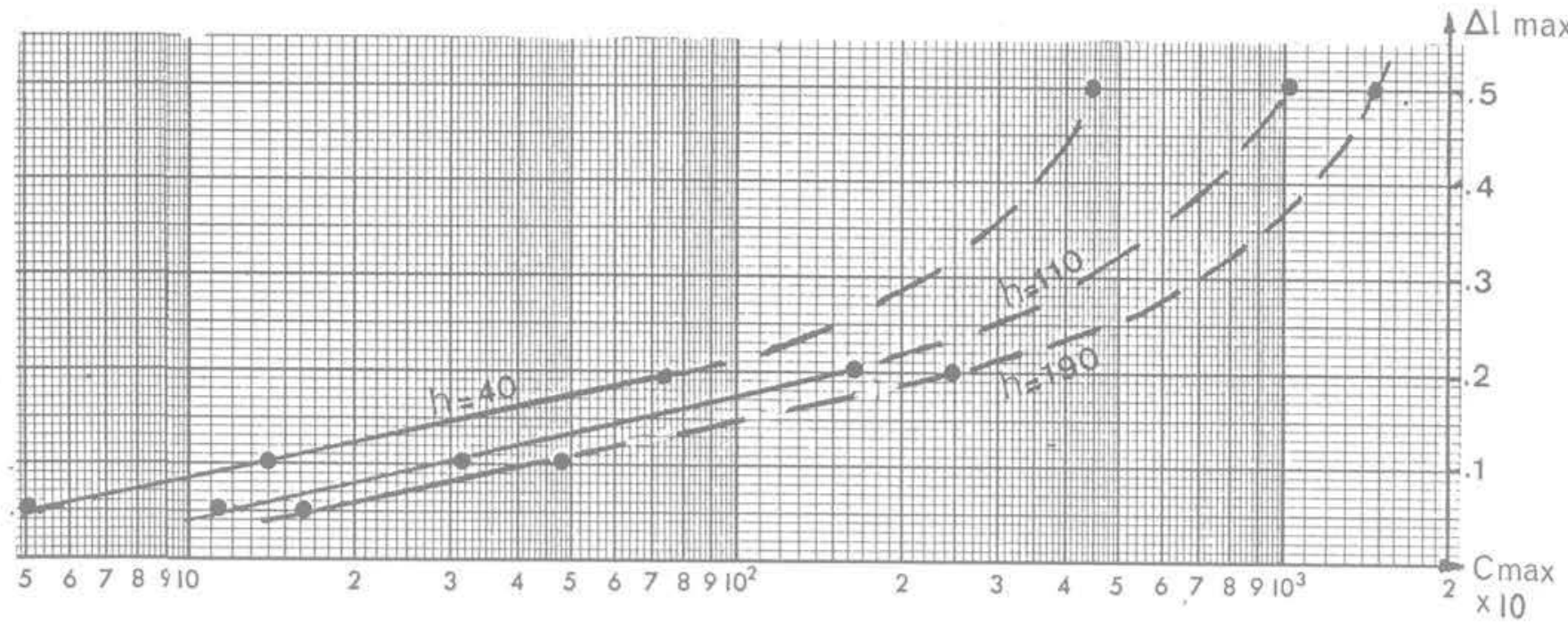

Fig. 15 Variation de l'amplitude Cmax avec Lmax pour quelques valeurs de "h»

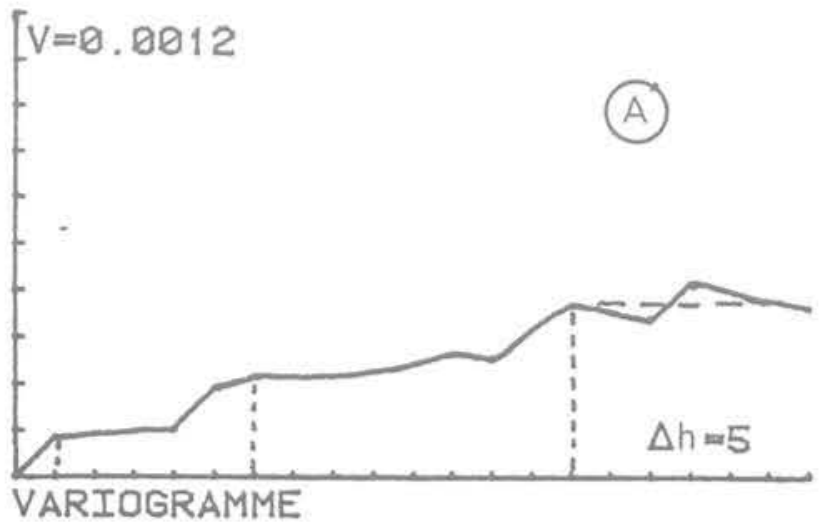

$\Delta \mathrm{Imax}=0.05-\mathrm{MAILLE}=5$

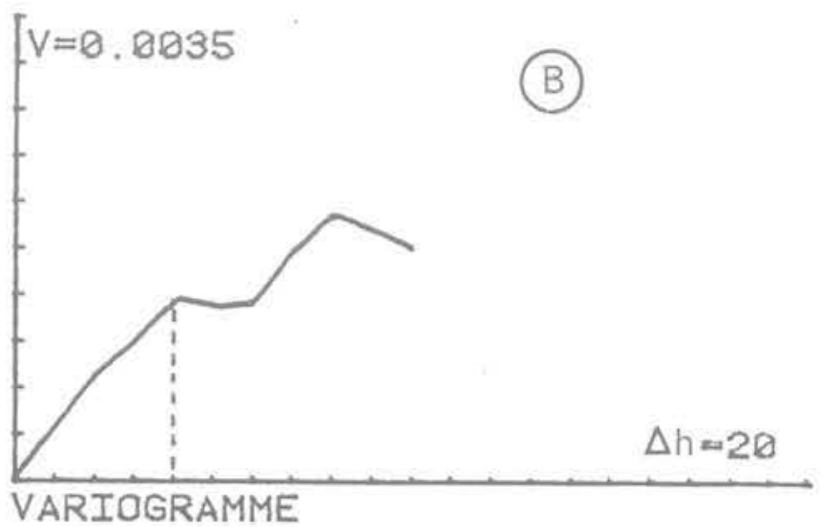

$\Delta \mathrm{Imax}=0.2-$ MAILLE $=20$

Fig. 16 Variogrammes du réseau YY750 aux mailles 5 et 20 


\section{Porosité de surface et porosité de volume}

Notre définition de la porosité fissurale linéaire ne diffère de la définition de F.H. Cornet que par la définition de la longueur de mesure $u_{0}$ et l'introduction de la notion géostatistique de maille de mesure optimale.

Elle ne modifie pratiquement pas la formulation utilisée par Cornet.

\subsection{Porosité de surface}

$$
f=f\left(\Omega, \Delta \ell, a_{0} n\right)=\frac{a_{0}}{a_{0}}
$$

qui s'exprime en fonction de la porosité linéaire par

$$
f\left(\Omega, \Delta \ell, a_{0} n\right)=\int_{0}^{2 \pi} \frac{\ell\left(\Omega, \Delta \ell, u_{0}\right) \cdot\left|u_{0}\right|^{2} d \theta}{\int_{0}^{2 \pi}\left|u_{0}\right|^{2} d \theta}
$$

où $n$ est le vecteur unitaire normal à la surface plane d'aire $a_{0}$.

En vertu de ce qui a été exposé $u_{0}$ ne dépend pas de $x$ en régime stationnaire mais dépend de $\theta$ sauf en cas d'isotropie (fig. 17 A).

\subsection{Porosité de volume}

La définition de Cornet s'écrira

$$
n(\Omega, \Delta \ell)=V_{0} / V_{0}
$$

où :

$$
\begin{array}{r}
V_{0}=\int_{0}^{\pi} d \theta \int_{0}^{2 \pi} d \varphi \int_{0}^{\theta u_{0} \mid}|u|^{2} \sin \theta|d u| \\
V_{0}^{P}=\int_{0}^{\pi} d \theta \int_{0}^{2 \pi} d \varphi \int_{0}^{\left|u_{0}\right|} 1\left(\Omega, \Delta \ell, u_{0}\right)|u|^{2} \sin \theta|d u|
\end{array}
$$

En coordonnées sphériques, $u_{0}$ dépend alors de $\varphi$ et $\theta$ mais pas de $x$ en régime stationnaire (fig. 17 B).

Notons que les calculs dans le plan et dans l'espace sont très simplifiés s'il existe un nombre limité de familles de fractures pour lesquelles on peut définir indépendamment les porosités normales partielles.

Par exemple, dans le plan, si l'on individualise les $\mathrm{k}$ porosités normales $\ell_{i}\left(u_{o i}\right)$ de $k$ familles (à partir d'une statistique sur leurs densités respectives) la valeur de $\ell_{i}(u)$ ne change pas avec l'angle $\theta$ si l'on fait subir à $u_{o i}$ la transformation:

$u_{o i}^{\prime}=u_{o i} \cos \theta_{i}$ dans le plan

$u_{o i}^{\prime}=u_{o i} \cos \theta_{1} \cos \Phi_{i}$ dans l'espace.

La porosité plane ou volumique s'écrit alors :

$$
f(\Omega, \Delta \ell)=\sum_{i=1}^{i=k} \ell\left(\Omega, \Delta \ell, u_{o i}^{\prime}\right)
$$

$\ell\left(\Omega, \Delta \ell, \mathrm{u}_{\mathrm{o}}^{\prime}\right)$ étant la porosité linéaire mesurée dans une direction quelconque recoupant les $k$ familles de fractures.

Dans chaque cas, $u_{0}$ est défini par un ensemble de $k$ vecteur $\mathrm{u}_{\mathrm{oi}}$.

L'analyse de la variance de la porosité plane ou spatiale nécessite dans le cas général une "montée sous puissance " qui n'est pas simple sauf dans le cas précédent d'un nombre fini de familles où son expression devient :

$$
\gamma\left(h^{\prime}\right)=\sum_{i=1}^{i-k} \gamma i\left(h^{\prime}\right)-\sum_{i=1}^{i-k} \sum_{i=1}^{i=k} C_{i j}\left(h^{\prime}\right)
$$

$h^{\prime}$ étant défini par :

$h^{\prime}=h / \cos \theta_{i}$ dans le plan

$h^{\prime}=h / \cos \theta_{i} \cos \Phi$ dans l'espace

$\mathrm{C}_{i j}\left(\mathrm{~h}^{\prime}\right)$ étant la covariance $\ell\left(\mathrm{x}, \mathrm{u}_{\mathrm{oi}}^{\prime}\right)$ avec $\ell\left(\mathrm{x}, \mathrm{u}_{\mathrm{o}}^{\prime}\right)$.

\section{Conclusion}

La porosité fissurale doit être analysée comme fonction d'une variable régionalisée vectorielle.

Elle peut être définie en probabilité dans un domaine stationnaire et peut alors prendre des valeurs différentes dans un même champ s'il y existe des structures définissant plusieurs domaines stationnaires en fonction de l'échelle d'investigation.

Cependant, nous avons dans ce qui précède considéré toute fracture comme un vide, ce qui n'est évidemment pas le cas général. La détermination de l'épaisseur moyenne des fractures n'est pas non plus un problème simple. Si l'on utilise une analyse de fracturation pour accéder à la porosité, il convient donc en général d'affecter à la porosité athéorique" de fissure un coefficient traduisant statistiquement le taux de remplissage de chaque famille de fissures. Un autre coefficient doit rendre compte des divergences entre porosité "optique " et porosité effective.

Mais il reste vrai que I'on pourra difficilement dans l'avenir concevoir des modèles sérieux en mécanique des roches et hydraulique des milieux fissurés sans aborder de front la caractérisation structurale du champ de porosité. Le développement efficient des méthodes numériques dans ces problèmes passe par cette analyse.

On notera, en outre, que s'il existe une relation entre la perméabilité des familles de fractures et leur orientation, l'analyse peut déboucher sur une régionalisation du tenseur de perméabilité.
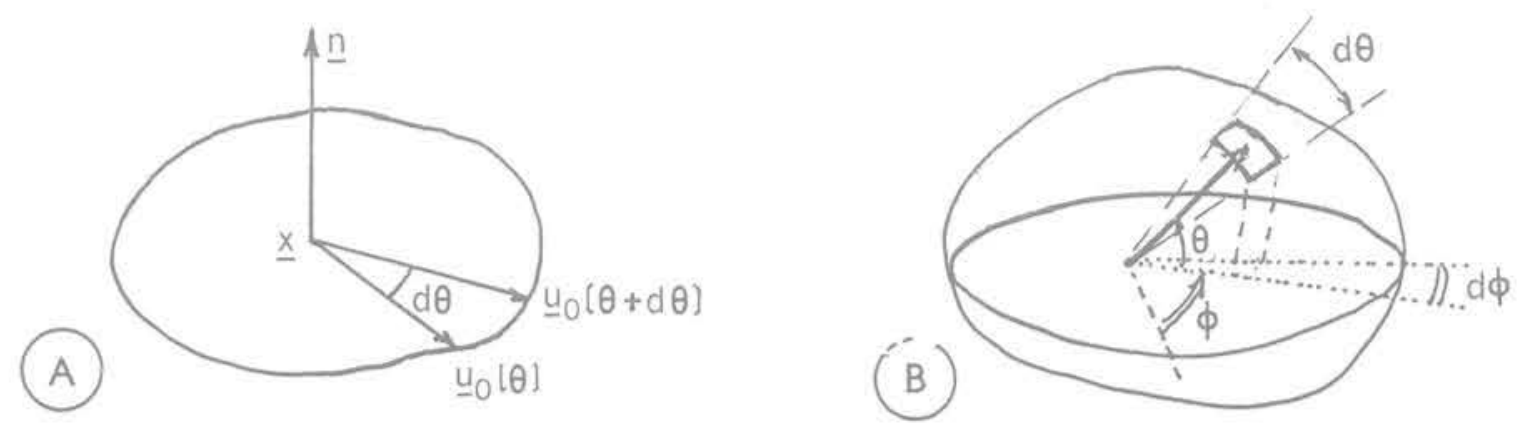

Fig. 17 Variation de $u_{0}(\theta)$ dans le plan, de $u_{0}(\varphi, \theta)$ dans l'espace 


\section{Références bibliographiques}

1 Cornet F.H. (1977) - "Étude du comportement élastique et fragile des roches saturées par un liquide. "Revue Fr. de Géotechnique $n^{\circ}$ 2; pp. 91-100.

2 Maas A., Matheron G., Serra J. (1967) - "Morphologie mathématique et granulométries en place. Annales des Mines; Nov. Déc. 1967; vol. XI, pp. 735753.

3 Serra (1967) - “ Un critère nouveau de découverte de structures: le variogramme.n. Sc. de la Terre; Tome XII, $n^{\circ} 4$, pp. 275-299.
4 Serra J. (1968) - "Les structures gigognes: morphologie mathématique et interprétation métallogénique." Mineral Deposita (Berlin); Vol. 3, pp. 135154.

5 Scheidegger A.E. (1964) - «The physics of flow through porous media. " Revised Edition, The MacMilIan comp; New York.

6 Thomas A., Therme P., Richard P. (1981) - "A new method for quick measuring and computing parameters of rock jointing. " C.r. $22^{e}$ US symposium of Rock Mechanics, Juin 1981. M.I.T. Cambridge, USA. 